\title{
The sharp version of a strongly starlikeness condition
}

\author{
Olga Engel \\ Babes-Bolyai University, \\ Cluj-Napoca, Romania \\ email: engel_olga@hotmail.com
}

\author{
Abdul Rahman S. Juma \\ University of Anbar, \\ Ramadi, Iraq \\ email: dr_juma@hotmail.com
}

\begin{abstract}
In this paper we give the best form of a strongly starlikeness condition. Some consequences of this result are deduced. The basic tool of the research is the method of differential subordinations.
\end{abstract}

\section{Introduction}

Let $\mathbb{U}=\{z \in \mathbb{C}:|z|<1\}$ be the open unit disk in the complex plane. Let $\mathcal{A}$ be the class of analytic functions $\mathrm{f}$, which are defined on the unit disk $\mathbb{U}$ and have the properties $f(0)=f^{\prime}(0)-1=0$. The subclass of $\mathcal{A}$, consisting of functions for which the domain $f(\mathbb{U})$ is starlike with respect to 0 is denoted by $S^{*}$. An analytic characterization of $\mathrm{S}^{*}$ is given by

$$
S^{*}=\left\{f \in \mathcal{A}: \operatorname{Re} \frac{z f^{\prime}(z)}{f(z)}>0, z \in \mathbb{U}\right\} .
$$

In connection with the starlike functions has been introduced the following class

$$
\mathrm{SS}^{*}(\alpha)=\left\{f \in \mathcal{A}:\left|\arg \frac{z f^{\prime}(z)}{\mathrm{f}(z)}\right|<\alpha \frac{\pi}{2}, \alpha \in(0,1], z \in \mathbb{U}\right\}
$$


which is the class of strongly starlike functions of order $\alpha$. Another subclass of $\mathcal{A}$ we deal with is the following

$$
\mathcal{G}_{\mathrm{b}}=\left\{f \in \mathcal{A}:\left|\frac{1+\frac{z f^{\prime \prime}(z)}{f^{\prime}(z)}}{\frac{z f^{\prime}(z)}{f(z)}}-1\right|<b, \quad z \in \mathbb{U}\right\},
$$

where $b>0$.

The authors of [3] proved the following result:

Theorem 1 If the function $\mathrm{f}$ belongs to the class $\mathcal{G}_{\mathrm{b}(\beta)}$ with

$$
b(\beta)=\frac{\beta}{\sqrt{(1-\beta)^{1-\beta}(1+\beta)^{1+\beta}}},
$$

where $0<\beta \leq 1$, then $f \in \operatorname{SS}^{*}(\beta)$.

Let $-1 \leq B<A \leq 1$. The class $S^{*}(A, B)$ is defined by the equality

$$
S^{*}(A, B)=\left\{f \in \mathcal{A}: \frac{z f^{\prime}(z)}{f(z)} \prec \frac{1+A z}{1+B z}, z \in \mathbb{U}\right\} .
$$

An other result regarding the class $\mathcal{G}_{\mathrm{b}}$ is the following theorem published in [4].

Theorem 2 Assume that $-1 \leq \mathrm{B}<\mathrm{A} \leq 1$ and $\mathrm{b}(1+|\mathrm{A}|)^{2} \leq|\mathrm{A}-\mathrm{B}|$. If $\mathrm{f} \in \mathcal{G}_{\mathrm{b}}$, then $\mathrm{f} \in \mathrm{S}^{*}(\mathrm{~A}, \mathrm{~B})$.

The aim of this paper is to prove the sharp version of Theorem 1, and an improvement of Theorem 2.

In our work we need the following results.

\section{Preliminaries}

Let $f$ and $g$ be analytic functions in $\mathbb{U}$. The function $f$ is said to be subordinate to $g$, written $f \prec g$, if there is a function $w$ analytic in $\mathbb{U}$, with $w(0)=0$, $|w(z)|<1, z \in \mathbb{U}$ and $f(z)=g(w(z)), z \in \mathbb{U}$. Recall that if $g$ is univalent, then $f \prec g$ if and only if $f(0)=g(0)$ and $f(\mathbb{U}) \subset g(\mathbb{U})$. 
Lemma 1 [1] Let $\mathrm{p}(z)=\mathrm{a}+\sum_{\mathrm{k}=\mathrm{n}}^{\infty} \mathrm{a}_{\mathrm{k}} z^{\mathrm{k}}$ be analytic in $\mathbb{U}$ with $\mathrm{p}(z) \not \equiv \mathrm{a}, \mathrm{n} \geq 1$ and let $\mathrm{q}: \mathbb{U} \rightarrow \mathbb{C}$ be an analytic and univalent function with $\mathrm{q}(0)=\mathrm{a}$. If $\mathrm{p}$ is not subordinate to $\mathrm{q}$, then there are two points $z_{0} \in \mathbb{U},\left|z_{0}\right|=r_{0}$ and $\zeta_{0} \in \partial \mathbb{U}$ and a real number $\mathrm{m} \in[\mathrm{n}, \infty)$, so that $\mathrm{q}$ is defined in $\zeta_{0}, \mathrm{p}\left(\mathbb{U}\left(0, \mathrm{r}_{0}\right)\right) \subset \mathrm{q}(\mathbb{U})$, and:

(i) $\mathrm{p}\left(z_{0}\right)=\mathrm{q}\left(\zeta_{0}\right)$,

(ii) $z_{0} p^{\prime}\left(z_{0}\right)=m \zeta_{0} q^{\prime}\left(\zeta_{0}\right)$,

(iii) $\operatorname{Re}\left(1+\frac{z_{0} p^{\prime \prime}\left(z_{0}\right)}{p^{\prime}\left(z_{0}\right)}\right) \geq m \operatorname{Re}\left(1+\frac{\zeta_{0} q^{\prime \prime}\left(\zeta_{0}\right)}{q^{\prime}\left(\zeta_{0}\right)}\right)$.

We note that $z_{0} p^{\prime}\left(z_{0}\right)$ is the outward normal to the curve $\mathrm{p}\left(\partial \mathbb{U}\left(0, r_{0}\right)\right)$ at the point $\mathrm{p}\left(z_{0}\right)$, while $\partial \mathbb{U}\left(0, r_{0}\right)$ denotes the border of the disc $\mathbb{U}\left(0, r_{0}\right)$.

A basic result we need in our research is the following:

Lemma 2 If $\mathrm{f} \in \mathcal{A}, \mathrm{b} \in[0,1)$, and $\mathrm{p}(z)=\frac{z \mathrm{f}^{\prime}(z)}{\mathrm{f}(z)}$, then the inequality

$$
\left|\frac{z p^{\prime}(z)}{p^{2}(z)}\right|<b, \quad z \in \mathbb{U}
$$

implies that

$$
p(z) \prec \frac{1}{1-b z} .
$$

The result is sharp.

Proof. If the subordination $\mathrm{p}(z) \prec \mathrm{q}(z)=\frac{1}{1-\mathrm{b} z}$ does not holds, then there are two points $z_{0} \in \mathbb{U},\left|z_{0}\right|=r_{0}<1$ and $\zeta_{0} \in \partial \mathbb{U}$ and a real number $m \in[1, \infty)$, so that $q$ is defined in $\zeta_{0}, p\left(\mathbb{U}\left(0, r_{0}\right)\right) \subset q(\mathbb{U})$, and:

$$
\begin{gathered}
\mathrm{p}\left(z_{0}\right)=\mathrm{q}\left(\zeta_{0}\right)=\frac{1}{1-\mathrm{b} \zeta_{0}} \\
z_{0} \mathrm{p}^{\prime}\left(z_{0}\right)=\mathrm{m} \zeta_{0} \mathrm{q}^{\prime}\left(\zeta_{0}\right)=\mathrm{m} \frac{\mathrm{b} \zeta_{0}}{\left(1-\mathrm{b} \zeta_{0}\right)^{2}} .
\end{gathered}
$$

Thus we get

$$
\frac{z_{0} p^{\prime}\left(z_{0}\right)}{p^{2}\left(z_{0}\right)}=m b \zeta_{0}
$$

Since $\left|m b \zeta_{0}\right| \geq b$, it follows that the equality (3) contradicts (2), and the proof is done. 


\section{Main results}

The following theorem is the sharp version of Theorem 1 .

Theorem 3 If $\alpha \in(0,1)$, and $\mathrm{f} \in \mathcal{G}_{\mathrm{b}(\alpha)}$, where $\mathrm{b}(\alpha)=\sin \left(\alpha \frac{\pi}{2}\right)$, then $\mathrm{f} \in$ $\mathrm{SS}^{*}(\alpha)$. The result is sharp.

Proof. If we denote $p(z)=\frac{z g^{\prime}(z)}{g(z)}$, then the condition $f \in \mathcal{G}_{\mathrm{b}(\alpha)}$ becomes

$$
\left|\frac{z p^{\prime}(z)}{p^{2}(z)}\right|<b(\alpha), \quad z \in \mathbb{U}
$$

and according to Lemma 2 we get

$$
\mathrm{p}(z) \prec \mathrm{q}(z)=\frac{1}{1-\mathrm{b}(\alpha) z} .
$$

The domain $D=q(\mathbb{U})$ is symmetric with respect to the real axis and the boundary of $\mathrm{D}$ is the curve

$$
\Gamma=\left\{\begin{array}{l}
x(\theta)=\operatorname{Re} \frac{1}{1-b(\alpha) e^{i \theta}}=\frac{1-b(\alpha) \cos \theta}{1+b^{2}(\alpha)-2 b(\alpha) \cos \theta}, \\
y(\theta)=\operatorname{Im} \frac{1}{1-b(\alpha) e^{i \theta}}=\frac{b(\alpha) \sin \theta}{1+b^{2}(\alpha)-2 b(\alpha) \cos \theta},
\end{array} \quad \theta \in[-\pi, \pi] .\right.
$$

The subordination $\mathrm{p}(z) \prec \mathrm{q}(z)$ implies that $|\arg (\mathrm{p}(z))| \leq \arctan (M)$, where $M$ is the slope of the tangent line to the curve $\Gamma$ trough the origin. The equation of the tangent line is

$$
\frac{x-x(\theta)}{x^{\prime}(\theta)}=\frac{y-y(\theta)}{y^{\prime}(\theta)} .
$$

This tangent line crosses the origin if and only if

$$
\frac{x(\theta)}{x^{\prime}(\theta)}=\frac{y(\theta)}{y^{\prime}(\theta)}
$$

and this equation is equivalent to

$$
2 b(\alpha) \cos ^{2} \theta-\left(3 b^{2}(\alpha)+1\right) \cos \theta+b(\alpha)\left(b^{2}(\alpha)+1\right)=0 .
$$

After a short calculation we get $\cos \theta=b(\alpha)$ and this implies

$$
M=\frac{y^{\prime}(\theta)}{x^{\prime}(\theta)}=\frac{y(\theta)}{x(\theta)}=\frac{b(\alpha) \sin \theta}{1-b(\alpha) \cos \theta}=\frac{b(\alpha)}{\sqrt{1-b^{2}(\alpha)}} .
$$


Finally if we put $b(\alpha)=\sin \left(\alpha \frac{\pi}{2}\right)$, then it follows that $|\arg (p(z))|$

$<\arctan (M)=\arctan \frac{b(\alpha)}{\sqrt{1-b^{2}(\alpha)}}=\alpha \frac{\pi}{2}, z \in \mathbb{U}$.

Thus we have proved the implication

$$
\left|\frac{z p^{\prime}(z)}{p^{2}(z)}\right|<\sin \left(\alpha \frac{\pi}{2}\right) \Rightarrow|\arg (p(z))|<\arctan (M)=\alpha \frac{\pi}{2},
$$

and the proof is done.

Putting $\alpha=1$ in Theorem 3, we get the following starlikeness condition, which is the sharp version of Corollary 1 from [3].

Corollary 1 If $\mathrm{f} \in \mathcal{A}$ and

$$
\left|\frac{1+\frac{z f^{\prime \prime}(z)}{f^{\prime}(z)}}{\frac{z f^{\prime}(z)}{f(z)}}-1\right|<1, \quad z \in \mathbb{U}
$$

then $\mathrm{f} \in \mathrm{S}^{*}$.

For $\alpha=\frac{1}{2}$, we get the sharp version of Corollary 2 from [3].

Corollary 2 If $\mathrm{f} \in \mathcal{A}$ and

$$
\left|\frac{1+\frac{z f^{\prime \prime}(z)}{f^{\prime}(z)}}{\frac{z f^{\prime}(z)}{f(z)}}-1\right|<\frac{\sqrt{2}}{2}, \quad z \in \mathbb{U}
$$

then $\mathrm{f} \in \mathrm{SS}^{*}\left(\frac{1}{2}\right)$.

Theorem 4 If $\mathrm{f} \in \mathcal{G}_{\mathrm{b}}$ and $\mathrm{b}(1+\mathrm{A}-\mathrm{B}+|\mathrm{B}|)<\mathrm{A}-\mathrm{B}$, then $\mathrm{f} \in \mathrm{S}^{*}(\mathrm{~A}, \mathrm{~B})$.

Proof. Let $q, h: \mathbb{U} \rightarrow \mathbb{C}$ be the functions defined by

$$
\mathrm{q}(z)=\frac{1}{1-\mathrm{bz}}, \mathrm{h}(z)=\frac{1+\mathrm{Az}}{1+\mathrm{B} z} .
$$

According to Lemma 2 we have $p(z)=\frac{z f^{\prime}(z)}{f(z)} \prec q(z)$ which is equivalent to

$$
\mathrm{p}(\mathbb{U}) \subset \mathrm{q}(\mathbb{U}) .
$$


We will prove that $q(\mathbb{U}) \subset h(\mathbb{U})$. A simple calculation shows that the domains $q(\mathbb{U})$ and $h(\mathbb{U})$ are convex.

The border of the domain $q(\mathbb{U})$ is the curve

$$
\Gamma: \quad \mathrm{q}\left(e^{i \theta}\right)=\frac{1}{1-b e^{i \theta}}, \quad \theta \in[0,2 \pi],
$$

and the border of $h(\mathbb{U})$ is the curve

$$
\Delta: \quad h\left(e^{i \eta}\right)=\frac{1+A e^{i \eta}}{1+B e^{i \eta}}, \quad \eta \in[0,2 \pi] .
$$

The inequality $b(1+A-B+|B|)<A-B$ is equivalent to $\frac{b}{1-b}<\frac{A-B}{1+|B|}$.

This inequality implies

$$
\left|q\left(e^{i \theta}\right)-1\right|=\frac{b}{\mid 1-b e^{i \theta \mid}} \leq \frac{b}{1-b}<\frac{A-B}{1+|B|} \leq \frac{A-B}{\left|1+B e^{i \eta}\right|}=\left|h\left(e^{i \eta}\right)-1\right| .
$$

Thus we get

$$
\left|q\left(e^{i \theta}\right)-1\right|<\left|h\left(e^{i \eta}\right)-1\right|, \quad \text { for every } \theta, \eta \in[0,2 \pi] .
$$

Since $1 \in \mathrm{q}(\mathbb{U})$ and $1 \in \mathrm{h}(\mathbb{U})$, the inequality (6) implies that the curve $\Gamma$ is inside the curve $\Delta$.

This means that

$$
\mathrm{q}(\mathbb{U}) \subset \mathrm{h}(\mathbb{U})
$$

For example if we consider

$$
\mathrm{q}(z)=\frac{1}{1-0.6 z} \text { and } \mathrm{h}(z)=\frac{1+0.3 z}{1-0.5 z}
$$

and the inequality $b(1+A-B+|B|)<A-B$ is satisfied for $b=0.6, A=0.3$ and $B=-0.5$ then we obtain the following graphics:

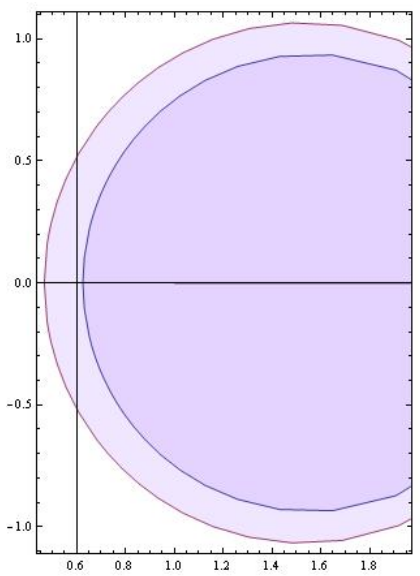


which shows that $q(\mathbb{U}) \subset h(\mathbb{U})$. For $b=0.7, A=0.3$ and $B=-0.5$ the inequality $\mathrm{b}(1+\mathrm{A}-\mathrm{B}+|\mathrm{B}|)<\mathrm{A}-\mathrm{B}$ is not satisfied and consequently we obtain the following image:

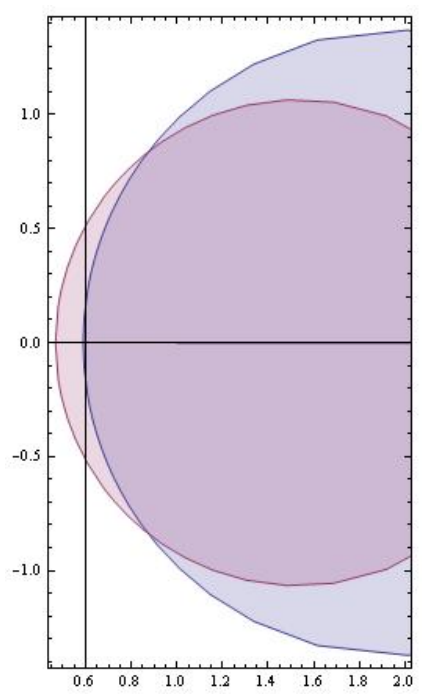

which shows that $q(\mathbb{U}) \not \subset h(\mathbb{U})$. Finally (5) and (7) implies $p(\mathbb{U}) \subset h(\mathbb{U})$ and since $h$ is univalent we infer $\frac{z f^{\prime}(z)}{f(z)}=p(z) \prec h(z), \quad z \in \mathbb{U}$.

This subordination is equivalent to $f \in S^{*}(A, B)$.

If $0 \leq \mathrm{B}<\mathrm{A} \leq 1$, then we get the following corollary, which improvs the result of Theorem 2 .

Corollary 3 Let $0 \leq B<A \leq 1$ and $\mathrm{b} \in(0,+\infty)$ such that $\mathrm{b}(1+\mathrm{A}) \leq 1+\mathrm{B}$. If $\mathrm{f} \in \mathcal{G}_{\mathrm{b}}$, then $\mathrm{f} \in \mathrm{S}^{*}(\mathrm{~A}, \mathrm{~B})$.

\section{References}

[1] S. S. Miller, P. T. Mocanu, Differential Subordinations. Theory and Applications, Marcel Dekker, New York, Basel 2000.

[2] S.S. Miller, P.T. Mocanu, The theory and applications of second-order differential subordinations, Stud. Univ. Babes-Bolyai Math., 34 (4) (1989), $3-33$. 
[3] M. Nunokawa, S. Owa, H. Saitoh, N. Takahashi, On a strongly starlikeness criteria, Bull. Inst. Math. Acad. Sinica, 31 (3) (2003), 195-199.

[4] J. Sokól, L. Trojnar-Spelina, On a sufficient condition for strongly starlikeness, J. Ineq. Appl., 383 (1) (2013).

Received: January 8, 2017 